\title{
Erratum to: Frontiers in Wastewater Treatment and Modelling
}

\author{
Giorgio Mannina ${ }^{(\bowtie)}$ \\ University of Palermo, Palermo, Italy \\ giorgio.mannina@unipa.it
}

\begin{abstract}
Erratum to:
Chapter "Energy Recovery from Immobilised Cells of Scenedesmus obliquus after Wastewater Treatment" in:

G. Mannina (ed.), Frontiers in Wastewater Treatment and Modelling, Lecture Notes in Civil Engineering 4, https://doi.org/10.1007/978-3-319-58421-8_42
\end{abstract}

The original version of the book was inadvertently published with misspelt author name "M. Gomes San Juan" in Chapter 'Energy Recovery from Immobilised Cells of Scenedesmus obliquus after Wastewater Treatment' which has to be corrected to read as "M. Gomez San Juan". The erratum chapter and the book have been updated with the change.

\section{Erratum to:}

Chapter "Study of the Competition Between Complete Nitrification by a Single Organism and Ammonia- and Nitrite-Oxidizing Bacteria" in: G. Mannina (ed.), Frontiers in Wastewater Treatment and Modelling, Lecture Notes in Civil Engineering 4, https://doi.org/10.1007/978-3-319-58421-8_45

In the original version of the book, the typographical error "Commamox" has been corrected to read as "Comammox" in Chap. 45, which is a belated correction.

The updated online version of these chapters can be found at https://doi.org/10.1007/978-3-319-58421-8

https://doi.org/10.1007/978-3-319-58421-8_42

https://doi.org/10.1007/978-3-319-58421-8_45

C) Springer International Publishing AG 2017

G. Mannina (ed.), Frontiers in Wastewater Treatment and Modelling,

Lecture Notes in Civil Engineering 4, https://doi.org/10.1007/978-3-319-58421-8_116 